\title{
Risk Factors for Procurence of Mucormycosis and its Manifestations Post Covid-19: a Single Arm Retrospective Unicentric Clinical Study
}

\author{
Semridhi $\operatorname{Gupta}^{1} \cdot$ Paridhi Ahuja $^{1,2}$ (I)
}

Received: 7 July 2021/Accepted: 14 August 2021/Published online: 18 September 2021

(C) Association of Otolaryngologists of India 2021

\begin{abstract}
To assess the risk factors causative of ROCM post COVID-19. To determine the clinical manifestations, causative factors leading to surge in ROCM \& factors contributing to poor prognosis in ROCM cases occurring in Western Rajasthan post COVID-19. In a Retrospective observational study, 56 post COVID-19 ROCM patients were analyzed from 'May-June 2021' at M.D.M. Hospital, Jodhpur. All patients after onset of disease were assessed as per selection criteria, included patients were examined for risk factors and clinical manifestations \& then were statistically evaluated. Median age was 53 years with male to female ratio 2.7:1. Uncontrolled diabetes was noted in at least $85 \%$ patients. Steroid use was seen in $66 \%$. Other major contributing factor to ROCM was the occupation; Out of 56 patients $69 \%$ were FARMERS.72\% had no history of COVID-19 vaccination. Only 3\% had no underlying disease. Overall mortality rate was $16 \% .2$ determinants were associated with poor prognosis: higher HbAlc-Out of 7 cases of orbital exenteration, $6(85 \%)$ had HbA1c $>12.36 \%$ took immoderate amounts of Steam inhalation. Maxillary sinuses were the most involved sites (94\%). Orbital involvement was seen in 33 and $12 \%$ were PL negative. Palatal involvement \& intracranial involvement were seen in $14 \%$ and $7 \%$ respectively. Bilateral
\end{abstract}

Paridhi Ahuja

paridhiahuja11@gmail.com

Semridhi Gupta

semridhigupta@gmail.com

1 Department of Otorhinolaryngology and Head and Neck Surgery, Dr. S.N. Medical College and M.D.M. Hospital, Jodhpur, Rajasthan 342003, India

2 PG Girls Hostel No. 03, Mathuradas Mathur Hospital, Jodhpur, Rajasthan 342003, India disease was seen in 28\%. Uncontrolled DM \& Humid working conditions came out to be the main predisposing factors for ROCM. Facial pain is considered to be the most common presenting complain. Maxillary sinus is the most involved site.

Keywords Rhino-orbital cerebral mucormycosis . Diabetes mellitus · Humidity · COVID-19 .

Facialpain and paraesthesia . Orbital involvement . Palatal involvement · Paranasal sinuses - Debridement . Maxillectomy $\cdot$ Eye exenteration

$\begin{array}{ll}\text { Abbreviations } \\ \text { ROCM } & \text { Rhino-orbital cerebral mucormycosis } \\ \text { DM } & \text { Diabetes Mellitus } \\ \text { MPS } & \text { Methylprednisolone }\end{array}$

\section{Introduction}

Coronavirus disease 2019 (COVID-19) is an infection caused by SARS CoV-2.Otorhinolaryngology has been a crucial part of this pandemic since starting, from nasopharyngeal swab sampling to anosmia becoming a typical symptom marker of COVID-19.In the recent times, we have come across yet another association between E.N.T. \& COVID-19 which is mucormycosis.

Mucormycosis is an acute, angioinvasive, and rapidly progressing disease caused by opportunistic fungal infection of the class Phycomycetes (order Mucorales) usually by Mucor or Rhizopus. The pathogen is ubiquitous, occurring naturally in the environment, the body surface, and orifices. Often in association with certain underlying 
diseases, that renders the body immunocompromised, the organism becomes invasive.

The ROCM type is the most common form of mucormycosis in India, followed by the pulmonary and cutaneous types whereas, the pulmonary form is the most common clinical presentation in developed countries [1].

The overall numbers of ROCM cases increased from 25 cases per year (1990-2007) to 89 cases per year (2013-2015) [2]. Another study from Tamil Nadu reported 9.5 cases per year during 2015-2019 [3]. Though it represents $8.3-13 \%$ of all fungal infections identified in autopsy, it still remained a rare infection but the present COVID-19 pandemic has witnessed a resurgence of cases of ROCM.

The first point that springs to one's mind when observing multiple patients of the same disease is the common factors that somehow link all the cases. The literature on causative factors of ROCM goes back to 1960's when Eugene et al. suggested diabetic ketoacidosis to be the most common underlying condition of ROCM.

Hariprasath et al. in his study showed that Uncontrolled diabetes mellitus is the most common underlying disease associated with mucormycosis in India, in contrast to haematological-malignancy patients and solid organ transplant recipients in developed countries [1].

And to move on from risk factors to the clinical features, the common complaints that these patients present with are facial pain, dental pain and discharging sinuses involving

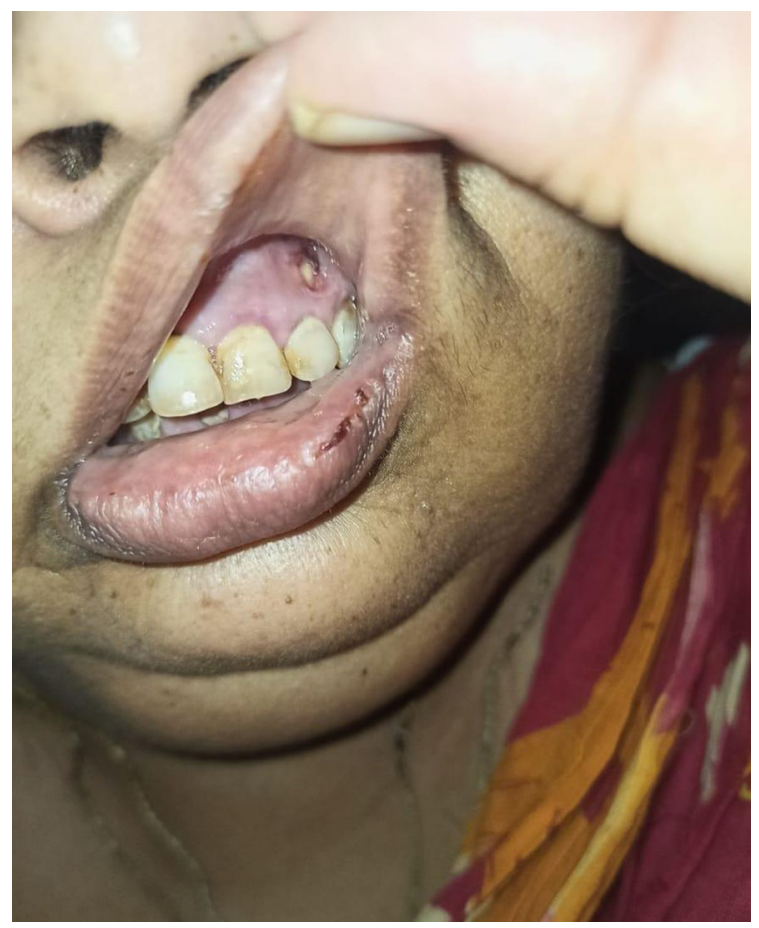

Fig. 1 Patient showing discharging sinus over upper alveolus the alveolus (Fig. 1), paraesthesia and swelling in malar region (Fig. 2), nasal discharge, periorbital swelling (Fig. 3) and headache.

And thus, the centre of our study is to find out the probable risk factors leading to ROCM, their presenting clinical and radiological manifestations in cases occurring in Western Rajasthan to enrich the literature that focuses on early detection of the disease and henceforth better prognosis.

\section{Methods}

This study was carried out at M.D.M Hospital to Dr. S.N. Medical College, Jodhpur, Rajasthan, India where all the cases of ROCM were managed; it included 56 ROCM positive patients admitted in the E.N.T. department in the month of May-June 2021. All the patients enrolled in the study were tested positive for ROCM by $\mathrm{KOH}$ mount of nasal biopsy or gingival biopsy or palatal scrapings.

Study design: Observational retrospective study.

Study location: Department of Oto-rhinolaryngology, M.D.M. Hospital, Dr. S.N. Medical College, Jodhpur, Rajasthan, India.

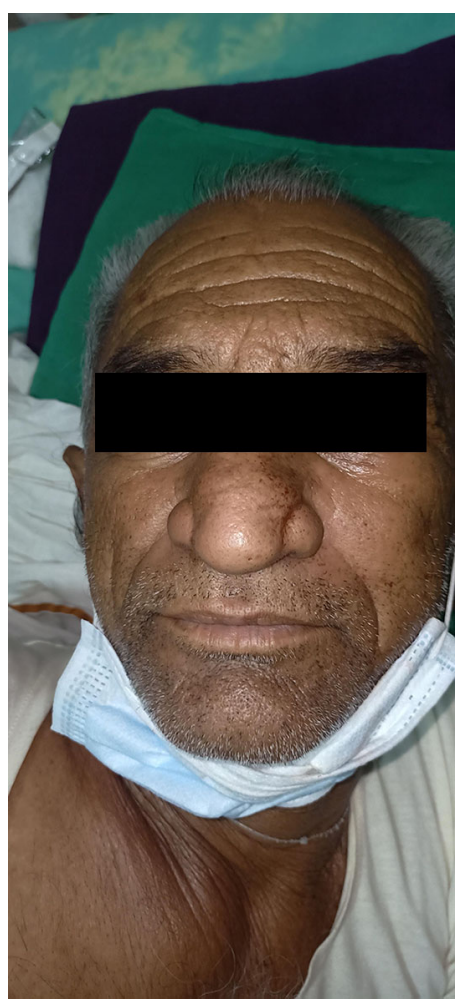

Fig. 2 Patient showing facial \& infraorbital swelling left side malar region 


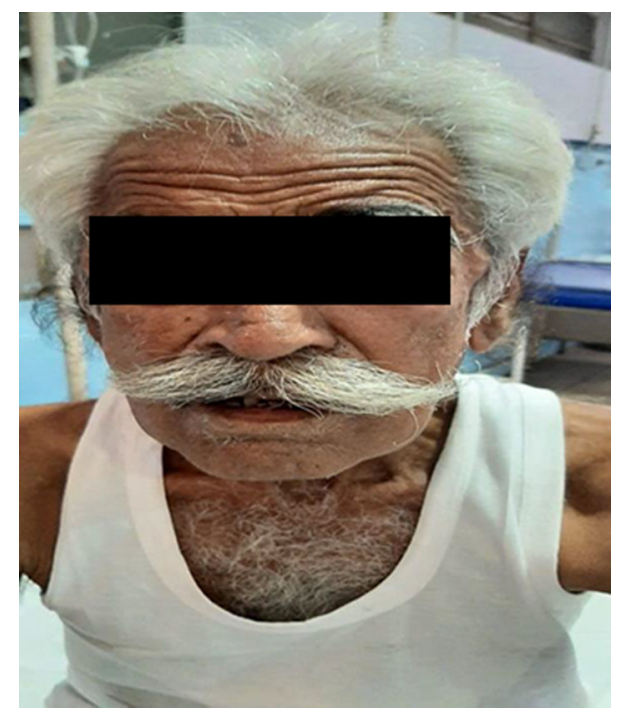

Fig. 3 Patient showing eye involvement

\section{Inclusion Criteria}

Patients who tested positive for mucormycosis by $\mathrm{KOH}$ mount of the nasal biopsy or gingival biopsy or palatal scrapings.

\section{Exclusion Criteria}

1. Patients tested positive for aspergillosis in $\mathrm{KOH}$ mount.

2. Patients who gave negative consent for admission \& further management.

\section{Methodology}

Data was collected by taking detailed history of patients regarding age, gender, occupation, presenting features and their duration, diabetic status, any other medical condition, history of covid-19 and associated management.

\section{Results}

The study included 56 patients of age $23-80$ years. Out of these 15 were females patients and 41 were males.

In our study, out of 56 patients 39 patients were farmers $(69 \%)$.

We also observed that most common underlying condition in patients developing ROCM was uncontrolled diabetes. 48 out of 56 patients (85\%) were diabetic and 39 of them were recently diagnosed. The mean HbA1c level was $8.5 \%$ with a range of $6.8-14.5 \%$.

Thirty seven patients in total had taken steroid treatment during covid-19 management. Out of these, 29 patients were given steroids by parenteral route and 8 of them were prescribed oral formulations.

Only 16 out of 56(28\%) had history of covid-19 vaccination. Out of these, only 2 patients got themselves vaccinated completely.

Twenty nine of them had other associated co-morbidities, bilateral pneumonitis (63\%) and hypertension (27\%) being most common.

Only three patients had no associated medical conditions.

Out of 56 patients, 9 patients (16\%) died within 15 days of surgical management and all of them were on $\mathrm{O} 2$ support pre operatively and were sent for ICU care post operatively.

Disease extension and hence the prognosis could be related to a few entities, HBa1c was $>12 \%$ in 6 out of 7 patients who had to undergo orbital exenteration. Around $36 \%$ patients with extensive disease took immoderate amounts of steam inhalation that hampers the mucociliary activity of nose (Table 1).

Table 1 Probable predisposing factors for mucormycosis

\begin{tabular}{lll}
\hline S. no & Risk factors & Number of patients involved \\
\hline 1 & Diabetes mellitus & 48 \\
2 & Farming occupation & 39 \\
3 & Steroid intake & 37 \\
4 & Non vaccinated for covid-19 & 40 \\
5 & B/L Pneumonitis & 18 \\
6 & Hypertension & 8 \\
7 & Steam inhalation & 7 \\
\hline
\end{tabular}




\section{DIABETES MELLITUS}

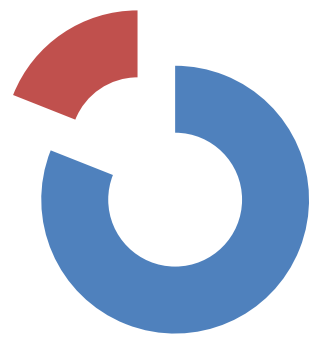
RECENTLY
DIAGNOSED
KNOWN CASES
OF DM TYPE 2

\section{Corelation between COVID-19 \& MUCORMYCOSIS}

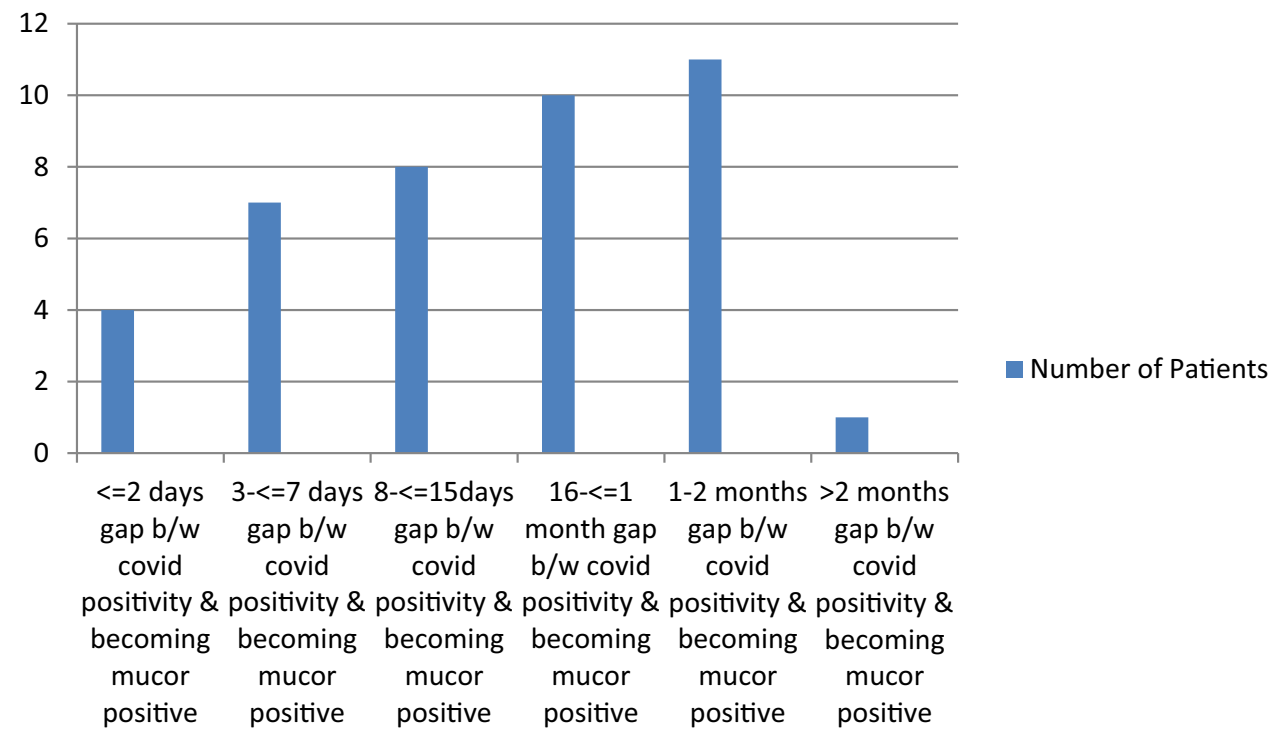

\section{PROGNOSTIC FACTORS}

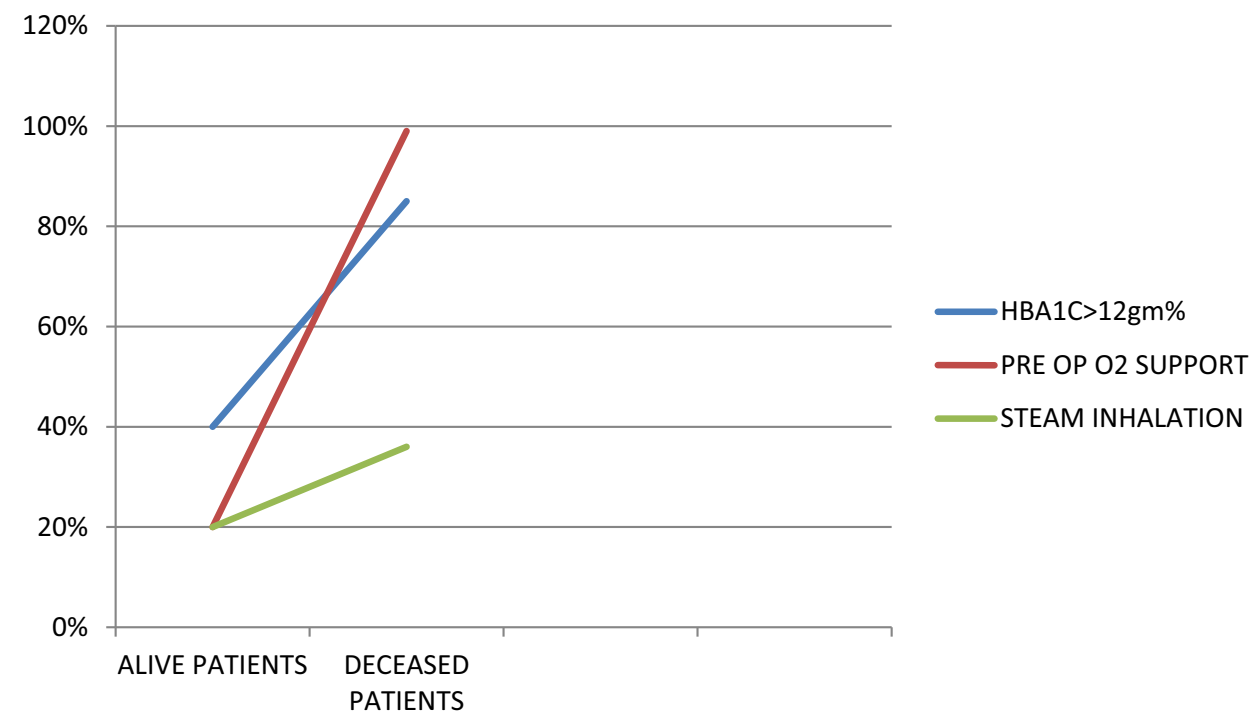




\title{
CLINICAL MANIFESTATIONS
}

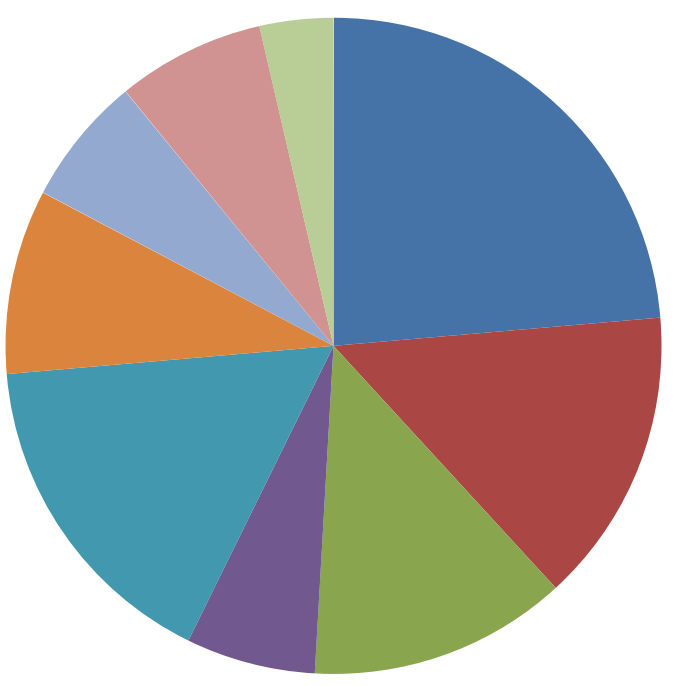

\author{
- FACIAL SWELLING \\ - CHEMOSIS \& DIMINUTION OF VISION \\ - PROPTOSIS \& PERIORBITAL SWELLING \\ - PERCEPTION OF LIGHT NEGATIVE \\ - HEADACHE \\ DENTAL PAIN \\ NASAL DISCHARGE \\ - PALATAL INVOLVEMENT \\ INTRACRANIAL INVOLVEMENT
}

Presenting Clinical features of these patients are summarized in the following pie chart.

Facial pain and swelling in malar region (26 patients) has been the most common presentation. Among the19 patients with ophthalmic involvement, chemosis and diminution of vision was the most frequent complaint (89\%) followed by proptosis and periorbital swelling (76\%), 7 of them were PL negative.18 patients experienced headache. 10 patients had onset of disease with dental pain. Seven of them had complains of bloody/blackish nasal discharge. Eight patients had shown maxilla involvement either in form of a discharging sinus or blackening of palate or loose tooth. Intracranial involvement was seen in four patients, along with the radiological evidence, one of them presented with hemiplegia and one with facial nerve palsy.

On radiological evaluation, maxillary sinuses were involved in $94 \%$ patients which is the most common site followed by ethmoids (79\%) and frontal (67\%) and sphenoid $(62 \%)$. Some of the cases presented with minimal disease and isolated maxillary sinus involvement was noted in $10 \%$. Bilateral disease seen in $28 \%$. Though maxillary sinuses were most frequently involved but bilaterality was more common in sphenoid sinuses (22\%) followed by maxillary sinuses (19\%). Intracranial involvement was seen in a total of four patients.

\section{- RADIOLOGICAL PRESENTATION OF ROCM}

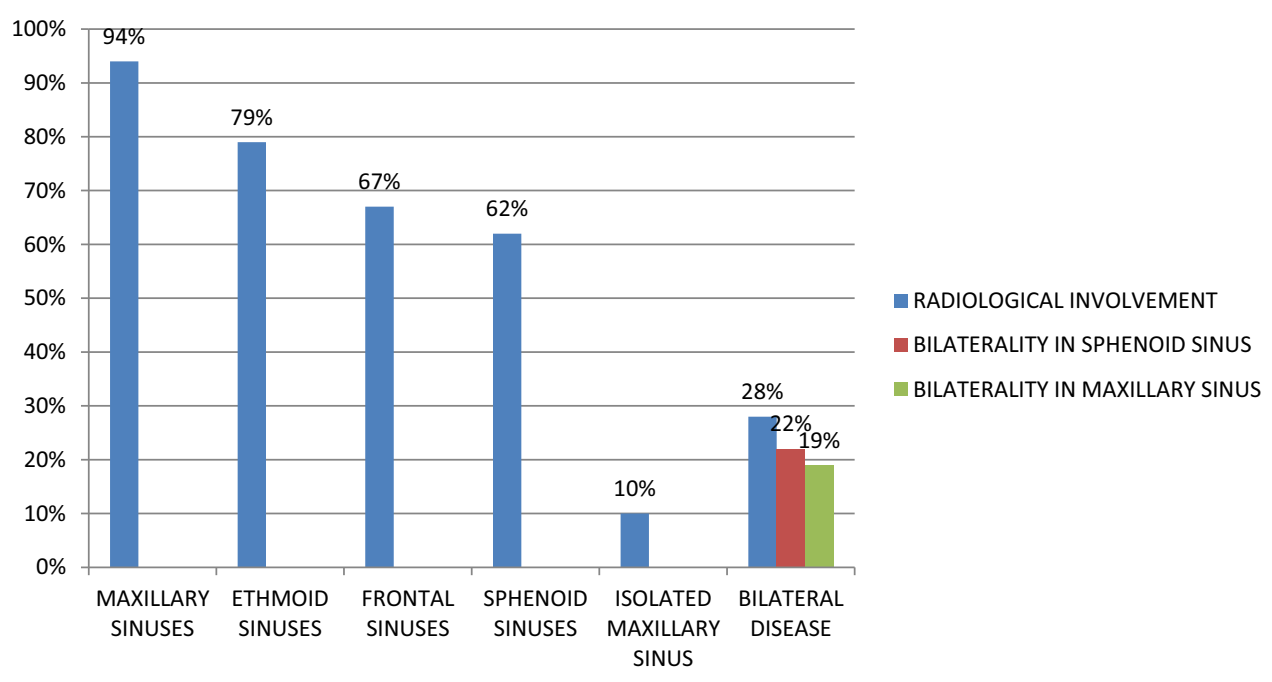




\section{NO. OF PATIENTS UNDERGOING VARIOUS TYPES OF SURGICAL INTERVENTION}

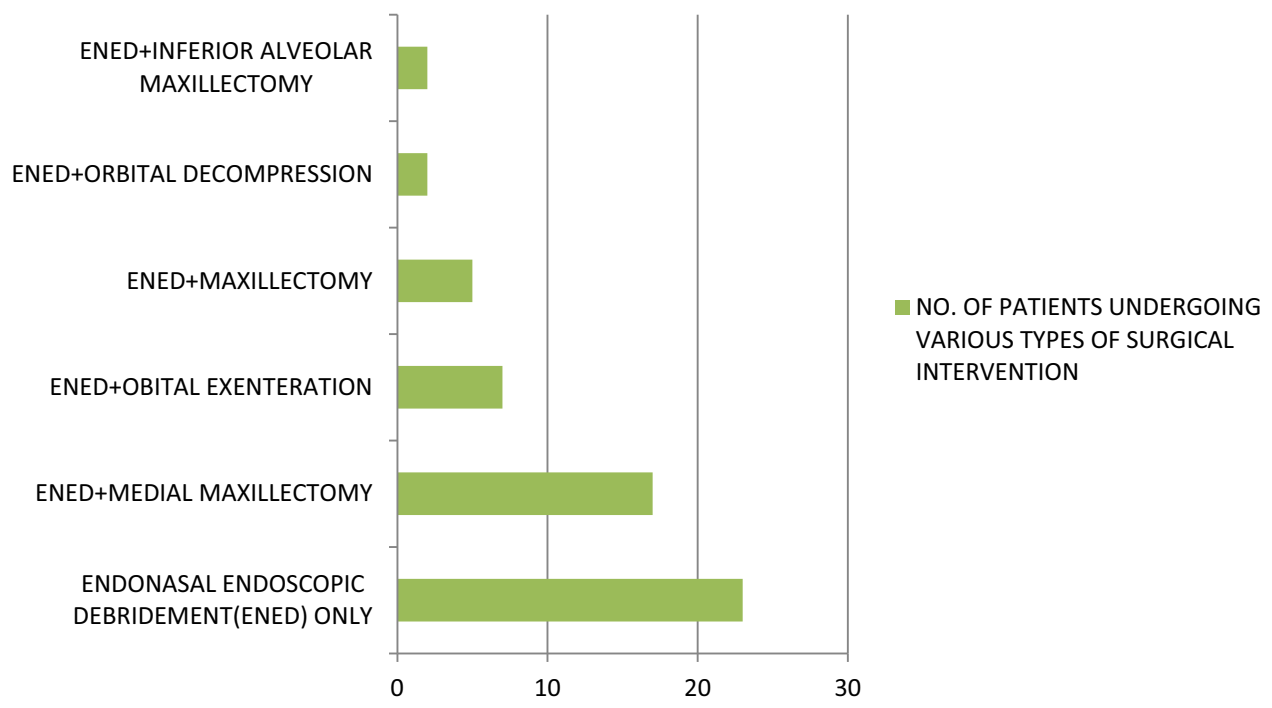

\section{Discussion}

A composite interaction of various factors including preexisting underlying medical conditions, largely diabetes mellitus type 2, previous respiratory compromise, use of immunosuppressive therapy, effect of covid-19 infection on the immunity has been implicated in the sudden increase in mucormycosis cases.

Our study shows that $85 \%$ of our patients had DM. Out of these majority (39) patients were recently diagnosed (either in our dept. or when admitted for covid-19 management). We took the liberty to describe our criteria 'recent' as within 2 months. This explains the uncontrolled blood sugar levels in them as these patients were unaware of their diabetic condition and few even developed ketoacidosis. Presence of diabetic ketoacidosis increases predisposition to mucormycosis. Acidosis disrupts iron binding of transferrin that leads to increased proportion of unbound iron, which may promote growth of the fungus.

Likewise, various other studies also conclude that DM is the most common predisposing factor aiding the occurrence of mucormycosis. In a study of 11 cases by Jiang et al. 9 out of 11 patients of mucormycosis had associated DM type2.Swati et al. concluded that the major risk factors for mucormycosis in the times of covid-19 included uncontrolled diabetes (96.7\%).According to a study by Chakrabarti et al. 53\% patients had associated DM. Patel et al. described 55\% of his patients had DM. Prakash et al. in his study pointed out $56 \%$ association with DM. According to Singh et al. $92 \%$ patients of mucormycosis had DM. And Patel et al. suggested this association to be $72 \%$ [3-9].

In our study the association between steroid intake and mucormycosis came out to be present in $66 \%$ patients. Parenterally, steroids were given in the form of Dexamethasone and Methylprednisolone whereas orally it was given in the form of Methylprednisolone. Those who were given injection MPS, average dose was $80 \mathrm{mg}$ for 8 days. Current guidelines in India recommend intravenous methylprednisolone $0.5-1 \mathrm{mg} / \mathrm{kg} / \mathrm{day}$ for three days in moderate cases and $1-2 \mathrm{mg} / \mathrm{kg} / \mathrm{day}$ in severe cases [10]. The guidelines specifically mention the risk of developing a secondary infection [11].

Many studies show association between steroid use and mucormycosis including Swati et al. which concludes that the major risk factors for mucormycosis in the times of covid-19 included concomitant steroid use in $61.2 \%$ patients, Jeong et al. described $33 \%$ association, Chakrabarti et al. says it to be associated in $8 \%$. Patel et al. in concluded this association to be $22 \%$ in 2017 and $3.7 \%$ in 2020, Prakash et al. reported $9.9 \%$ association, Singh et al. concluded $8 \%$ association.

There are few entities that came out to be showing positive correlation with mucormycosis in our study which have not been discussed much in other studies. One of them is the occupation of the patients, though a diverseness was seen but still when noted, majority of the patients were farmers $(69 \%)$. This can be attributed to the fact that in an otherwise dry state of Western Rajasthan, this profession provides quiet humid working conditions. 
A study conducted by El-Herte also ascribed fungal growth to the humidity. It says mucormycosis has a positive correlation with humidity [12].

COVID-19 has definitely been an accelerating factor for the surge of mucormycosis cases and though more detailed research work is needed to clam this but still our study describes a negative correlation between covid-19 vaccination and mucormycosis. Only 26 out of 56 patients got themselves vaccinated against covid-19, out of which only 2 patients give history of complete vaccination. Swati et al. in their study showed that $62 \%$ of their patients were covid19 positive. In our study while almost all the patients experienced covid-19 symptoms, only $73 \%$ give history of being COVID-19 positive as many of them didn't get themselves tested, and around $32 \%$ had b/l pneumonitis.

Apart from pre-op $\mathrm{O} 2$ dependency which would be due to the functional compromise of the lungs \& poor blood sugar control, another factor that according to our study contributed to poor prognosis was excessive steam inhalation, this can be attributed to its adverse effect on the mucociliary activity of nose and nasopharynx. as we talk about the presenting features, it is the clinical manifestations that the patients presented with to us in our department that has been mentioned in our study and used for statistical evaluation. Later on, in a lapse of 1-2 days, many patients' clinical picture got complicated and overlapped.

Patients with both ophthalmoplegia and PL negative eye were considered for orbital exenteration as ophthalmoplegia suggests infiltration of extraocular muscles.

Radiological investigations are important not to diagnose but to see the extent of the disease. CEMRI brain + orbit + pns is the investigation of choice. Paranasal sinuses were seen involved in all the patients with maxillary sinusitis being present in $94 \%$ cases followed by ethmoid sinus involvement in $79 \%$. Bhansali et al. also suggest $100 \%$ PNS involvement whereas Ferry et al. and Yohai et al. suggested $69 \%$ and $79 \%$ involvement respectively $[14,15]$.

Since most patients had no maxilla/palate involvement and no orbital complications, majority of patients were treated with endoscopic endonasal debridement alone. This can be credited to the fact that the patients were treated

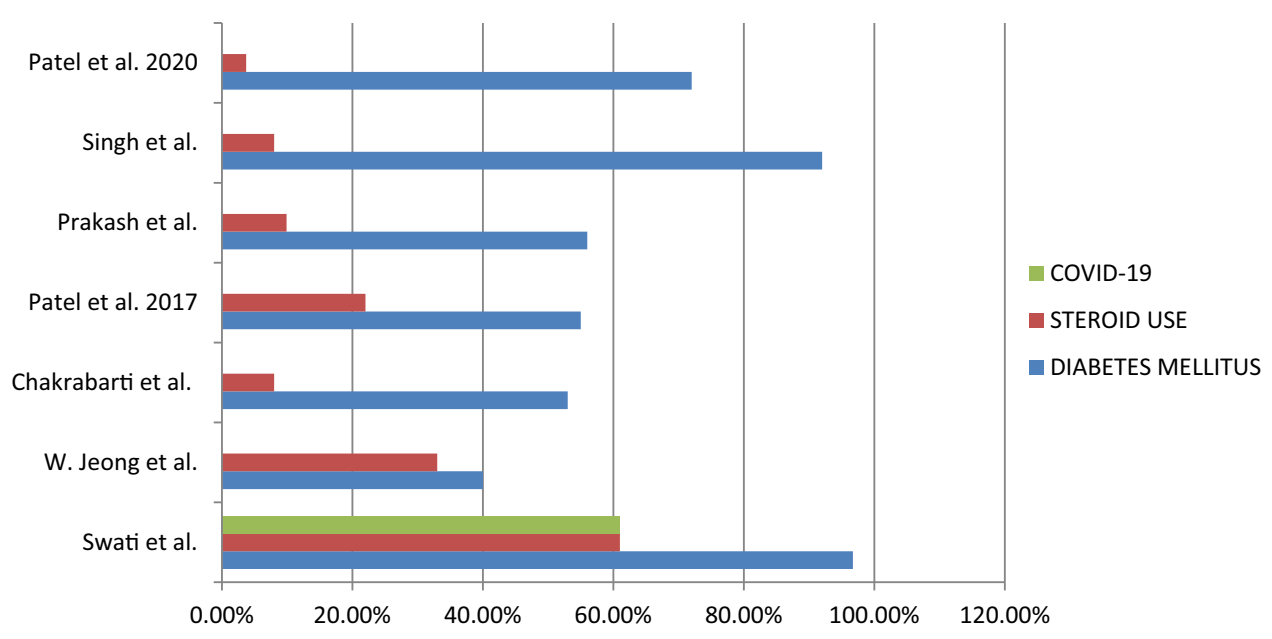

Facial swelling and orbital involvement was noted in 46 and $33 \%$ patients respectively by us, as compared to 46 and $89 \%$ respectively in a study done by Bhansali et al. Toothache and nasal discharge was noted in only 17 and $12 \%$ in our study whereas, Bhansali et al. showed this association to be 24 and $74 \%$. Headache and palatal involvement was seen in 18 and $14 \%$ of our patients; according to Bhansali et al. it is 20 and 29\% respectively. Among the ophthalmic manifestations, chemosis and diminution of vision were most common $(89 \%)$ followed by proptosis and periorbital swelling while Bhansali et al. suggest ophthalmoplegia (89\%) to be the leading clinical presentation followed by proptosis, loss of vision (80\%) and then chemosis (74\%) [13]. In our study, promptly long before the disease progression could occur. Also radical surgical procedures were attempted only when MRI or $\mathrm{KOH}$ mount advocated that eye or maxilla are involved and needs to be respected for complete clearance of disease.

\section{Conclusion}

In our study, it is seen that humid working conditions contributed to sudden rise of ROCM cases as majority of these were farmers and correlation between humidity \& fungal growth is a well known and established fact. DM 
type 2 was the most consistent risk factor. Among all these patients, majority had recently diagnosed diabetes which explains the uncontrolled blood sugar levels. This might have lead to acidosis in these patients acidosis disrupts iron binding property of transferrin that leads to increased proportion of unbound iron, which may promote growth of the fungus. These patients had acquired DM type 2 mostly during the COVID-19 management \& according to our assessment rarely any patient developed ROCM after 2-3 months gap of COVID-19. This also explains that average $\mathrm{HbA} 1 \mathrm{C}$ was noted $8.5 \mathrm{gm} \%$. Significant rise in $\mathrm{HbA} 1 \mathrm{C}$ is seen after $2-3$ months. Patients in initial stages presented with facial pain/swelling and periorbital swelling /mild headache/ nasal discharge. Those with progressive disease had severe ophthalmic involvement, palatal involvement, intracranial involvement. CEMRI brain + orbit + pns was useful to describe disease extension, to differentiate between normal mucosal thickenings, sinusitis changes and fungal components. Maxillary sinuses were most commonly involved.

\section{Declarations}

Conflict of interest Authors Semridhi Gupta and Paridhi Ahuja declare that they have no conflict of interest.

\section{References}

1. Prakash H, Chakrabarti A (2021) Epidemiology of Mucormycosis in India. Microorganisms 9(3):523. https://doi.org/10.3390/ microorganisms 9030523

2. Manesh A, Rupali P, Sullivan MO et al (2019) Mucormycosis-a clinicoepidemiological review of cases over 10 years. Mycoses 62:391-398. https://doi.org/10.1111/myc.12897

3. Prakash H, Anup Kumar Ghosh, Shivaprakash Mandya Rudramurthy, Pankaj Singh, Immaculata Xess, Jayanthi Savio, Umabala Pamidimukkala, Joseph Jillwin, Subhash Varma, Ashim Das, Naresh K Panda, Surjit Singh, Amanjit Bal, Arunaloke Chakrabarti (2019) A prospective multicenter study on mucormycosis in India: Epidemiology, diagnosis, and treatment. Med Mycol 57(4):395-402. https://doi.org/10.1093/mmy/ myy060

4. Jiang N, Zhao G, Yang S et al (2016) A retrospective analysis of eleven cases of invasive rhino-orbito-cerebral mucormycosis presented with orbital apex syndrome initially. BMC Ophthalmol 16:10. https://doi.org/10.1186/s12886-016-0189-1
5. Ravani Swati A, Agrawal Garima A, Leuva Parth A, Modi Palak H, Amin Krisha D (2021) Rise of the phoenix. Indian J Ophthalmol 69(6):1563-1568. https://doi.org/10.4103/ijo.IJO_ 310_21

6. Chakrabarti A, Das A, Mandal J, Shivaprakash MR, George VK, Tarai B, Rao P, Panda N, Verma SC, Sakhuja V (2006) The rising trend of invasive zygomycosis in patients with uncontrolled diabetes mellitus. Med Mycol 44(4):335-342. https://doi.org/ 10.1080/13693780500464930 (PMID: 16772227)

7. Chakrabarti A, Chatterjee SS, Das A, Panda N, Shivaprakash MR, Kaur A, Varma SC, Singhi S, Bhansali A, Sakhuja V (2009) Invasive zygomycosis in India: experience in a tertiary care hospital. Postgrad Med J 85(1009):573-581. https://doi.org/ 10.1136/pgmj.2008.076463 (PMID: 19892892)

8. Singh VP, Bansal C, Kaintura M (2019) Sinonasal mucormycosis: A to Z. Indian J Otolaryngol Head Neck Surg 71(Suppl 3):1962-1971. https://doi.org/10.1007/s12070-018-1384-6. Epub 2018 May 4. PMID: 31763277; PMCID: PMC6848679

9. Patel A, Kaur H, Xess I, Michael JS, Savio J, Rudramurthy S, Singh R, Shastri P, Umabala P, Sardana R, Kindo A, Capoor MR, Mohan S, Muthu V, Agarwal R, Chakrabarti A (2020) A multicentre observational study on the epidemiology, risk factors, management and outcomes of mucormycosis in India. Clin Microbiol Infect 26(7):944.e9-944.e15. https://doi.org/10.1016/ j.cmi.2019.11.021

10. Clinical management protocol for COVID-19. (2020) Accessed: July 7, 2020:https://www.mohfw.gov.in/pdf/ClinicalManagement ProtocolforCOVID19.pdf.

11. COVID-19 Treatment Guidelines Panel. Coronavirus disease 2019 (COVID-19) treatment guidelines. National Institutes of Health. (2020).Accessed:July 7， 2020: https://www.covid19 treatmentguidelines.nih.gov/.

12. El-Herte RI, Baban TA, Kanj SS (2012) Mucormycosis: a review on environmental fungal spores and seasonal variation of human disease. Adv Infectious Dis 2(3):76-81. https://doi.org/10.4236/ aid.2012.23012

13. Bhansali A, Bhadada S, Sharma A et al (2004) Presentation and outcome of rhino-orbital-cerebral mucormycosis in patients with diabetes. Postgrad Med J 80:670-674

14. Yohai RA, Bullock JD, Aziz AA, Markert RJ (1994) Survival factors in rhino-orbital-cerebral mucormycosis. Surv Ophthalmol 39(1):3-22. https://doi.org/10.1016/s0039-6257(05)80041-4. PMID: 7974189.

15. Ferry AP, Abedi S (1983) Diagnosis and management of rhinoorbitocerebral mucormycosis (phycomycosis). A report of 16 personally observed cases. Ophthalmology 90(9):1096-104. https://doi.org/10.1016/s0161-6420(83)80052-9. 6646648 .

Publisher's Note Springer Nature remains neutral with regard to jurisdictional claims in published maps and institutional affiliations. 